\title{
Subject Specific Finite Element Modelling of the Levator Ani
}

\author{
Su-Lin Lee ${ }^{1,2}$, Ara Darzi ${ }^{2}$, and Guang-Zhong Yang ${ }^{1,2}$ \\ ${ }^{1}$ Royal Society/Wolfson Foundation Medical Image Computing Laboratory, \\ ${ }^{2}$ Department of Surgical Oncology and Technology, \\ Imperial College London, London, United Kingdom \\ \{su-lin.lee, a.darzi, g.z.yang\}@imperial.ac.uk
}

\begin{abstract}
Understanding of the dynamic behaviour of the levator ani is important to the assessment of pelvic floor dysfunction. Whilst shape modelling allows the depiction of 3D morphological variation of the levator ani between different patient groups, it is insufficient to determine the underlying behaviour of how the muscle deforms during contraction and strain. The purpose of this study is to perform a subject specific finite element analysis of the levator ani with open access magnetic resonance imaging. The method is based on a Mooney-Rivlin hyperelastic model and permits dynamic study of subjects under natural physiological loadings. The value of the proposed modelling framework is demonstrated with dynamic 3D data from nulliparous, female subjects.
\end{abstract}

\section{Introduction}

The levator ani is a tripartite, striated muscle group of the pelvic floor and consists of the pubococcygeus, the iliococcygeus and the puborectalis. Childbirth has been shown to be one of the primary causes of injury to the levator ani with symptoms ranging from pain or constipation to faecal or urinary incontinence [1]. In current clinical settings, diagnoses are made either with full clinical examinations or using magnetic resonance imaging (MRI) in mid-sagittal and mid-coronal planes with measurements made from the position of organs to the pubococcygeal line [2], and an evacuation scan is popular with this method. Treatments for pelvic floor dysfunction include pelvic floor exercises and surgery. The usual surgical treatment of the pelvic floor is divided between the urologist, gynaecologist and proctologist. Existing research has shown that among women who have had surgery, approximately $20 \%$ require a second operation. This is partially due to the current surgical practice and the general lack of quantitative assessment in guiding the surgical procedures. A more unified way of understanding the levator ani in 3D, as well as the location of any injuries is essential for reducing the rate of repeated operation.

Hoyte, et al, [3,4] initiated the use of 3D models of the levator ani in asymptomatic and symptomatic women. Each shape was manually segmented and the groups were compared by measurements. Lee et al. extended 3D modelling of the levator ani with statistical shape models that allowed the identification of the main modes of variation of the shape of the levator in normal subjects at rest, on maximal contraction, and on 
maximal strain [5,6]. Whilst these techniques provide the visualisation and understanding of the 3D morphology of the levator, they do not provide an insight into how the muscle strains during the performance of pelvic floor exercises.

To elucidate dynamic behaviour of the soft tissue, finite element models (FEM) have been widely used to model biological tissues such as bone, myocardium, brain deformation and breast tissue deformation. For example, Pathmanathan, et al. [7] have modelled breast deformation when the patient is standing, supine and compressed during mammography. d'Aulignac, et al, [8] used shell elements (thinwalled structures) to build a FEM of the levator ani. The model took into account the direction of the muscle fibres and the incompressibility of the tissue, and used geometrical data obtained by Janda, et al, [9-11], where morphological parameters of the levator ani, including geometry of the muscle fibres and muscle sarcomere length, are measured from cadavers. Force-length curves, which determine the maximal force in certain positions, were derived and the material properties of the muscle were investigated by using $20 \mathrm{~mm}^{2}$ samples of the cadaver tissue to determine the biaxial stress-strain [10].

In this study, a FEM based on patient specific data, rather than idealised models with a constant thickness throughout the muscle sheet, is used to predict dynamic properties of the pelvic floor under different physiological loadings. Pelvic floor exercise involves extensive tissue deformation during maximal contraction or maximal strain. Linear models are inappropriate in this case as linear constitutive laws, such as Hooke's law, do not hold. A Mooney-Rivlin hyperelastic model is used instead in this study. The value of the proposed modelling framework is demonstrated with dynamic $3 \mathrm{D}$ data from three nulliparous, female subjects using open access MRI.

\section{Methods}

\subsection{Hyperelasticity}

Hyperelasticity is a classical modelling framework that is applicable to biological tissues. It can account for both mechanical and geometric non-linearities in the mathematical formulation. A material is considered elastic when the stress $\mathrm{S}$ at a point $\mathrm{X}$ depends on the deformation gradient $\mathrm{F}$. A material is considered hyperelastic when the stress $\mathrm{S}$ can be derived from both the deformation gradient $\mathrm{F}$ and a strain energy function $\mathrm{W}$ :

$$
S=\frac{\partial W}{\partial E}
$$

where $\mathrm{E}$ is the Lagrangian strain tensor, defined as:

$$
\begin{aligned}
& E^{(m)}=\frac{1}{m}\left(U^{m}-I\right), \quad m \neq 0 \\
& E^{(0)}=\ln U, \quad m=0 .
\end{aligned}
$$

where $\mathrm{U}$ is the right stretch tensor and is obtained from the deformation gradient $\mathrm{F}$ by polar decomposition: 


$$
F=R U
$$

In Eq. (3), $\mathrm{R}$ is a proper orthogonal tensor and $\mathrm{U}$ is positive definite and symmetric.

As it is practically difficult to test the muscle material of a levator ani in vivo, a five parameter Mooney-Rivlin hyperelastic material model was adopted with the parameters from the work by [12]: $\mathrm{c}_{10}=2.5 \mathrm{kPa}, \mathrm{c}_{20}=0.625 \mathrm{kPa}, \mathrm{c}_{01}=0, \mathrm{c}_{02}=0, \mathrm{c}_{11}=$ 0 . Such models have been used to model the human tongue (and in turn was originally developed for the myocardium and other general soft tissues) and facial muscles [13]. The Mooney-Rivlin model approximates the energy function W:

$$
\begin{aligned}
W & =c_{10}\left(I_{1}-3\right)+c_{01}\left(I_{2}-3\right)+c_{20}\left(I_{1}-3\right)^{2} \\
& +c_{11}\left(I_{1}-3\right)\left(I_{2}-3\right)+c_{02}\left(I_{2}-3\right)^{2}
\end{aligned}
$$

where $I_{1}$ and $I_{2}$ are the first and second invariants of the strain tensor $E$. An examination of a square centimetre of the material shows that stretch causes a reduction in width in the direction perpendicular to the stretch.

\subsection{Magnetic Resonance Image Acquisition}

The anatomical structure of the levator ani during different levels of stress were built from magnetic resonance images acquired from a 0.5T GE iMR open access scanner. Each 3D data set was acquired with a turbo spin echo sequence (TR $=4900 \mathrm{~ms}$, TE $=$ $102 \mathrm{~ms}$, slice thickness $=5.5 \mathrm{~mm}$ ) for each of 3 nulliparous, female subjects with a total scan time of approximately 5 minutes. The ages for Subjects 1, 2 and 3 were 24, 31 and 23 , respectively.

\subsection{Finite Element Modelling}

For this study, dynamic 3D morphological segmentation of the levator ani was based on the work by Lee et al [6]. The node correspondence for each levator ani surface was automatically determined by using a harmonic embedding framework developed by Horkaew et al [14]. The optimisation also took into account the thickness information at each node, calculated to be the minimal distance from the bottom surface of the levator to its corresponding top surface. Each shape is defined by a total of 2401 nodes (with a specific thickness at each node) and 4608 elements. Each levator ani was subsequently modelled using SHELL181 elements in ANSYS with the nodes in the areas connected to the pelvic bone, ligament and coccyx fixed in translation but left free to rotate. There are no fixed degrees of freedom on all other nodes. Thickness information was added at each node. Pressures were applied to each surface to simulate contraction and strain of the levator ani.

\section{Results}

The morphological change for each of the three subjects can be seen in Figure 1. The shape variation associated with contraction and strain is consistent with the expected 
anatomical change. The contraction shows a lifting of the levator ani while the strain leads to a downward movement and spread of the levator ani wings. The von Mises stress for each surface is displayed in Figure 2, where the stress distribution is consistent with areas involving the most significant movement during pelvic floor exercise.
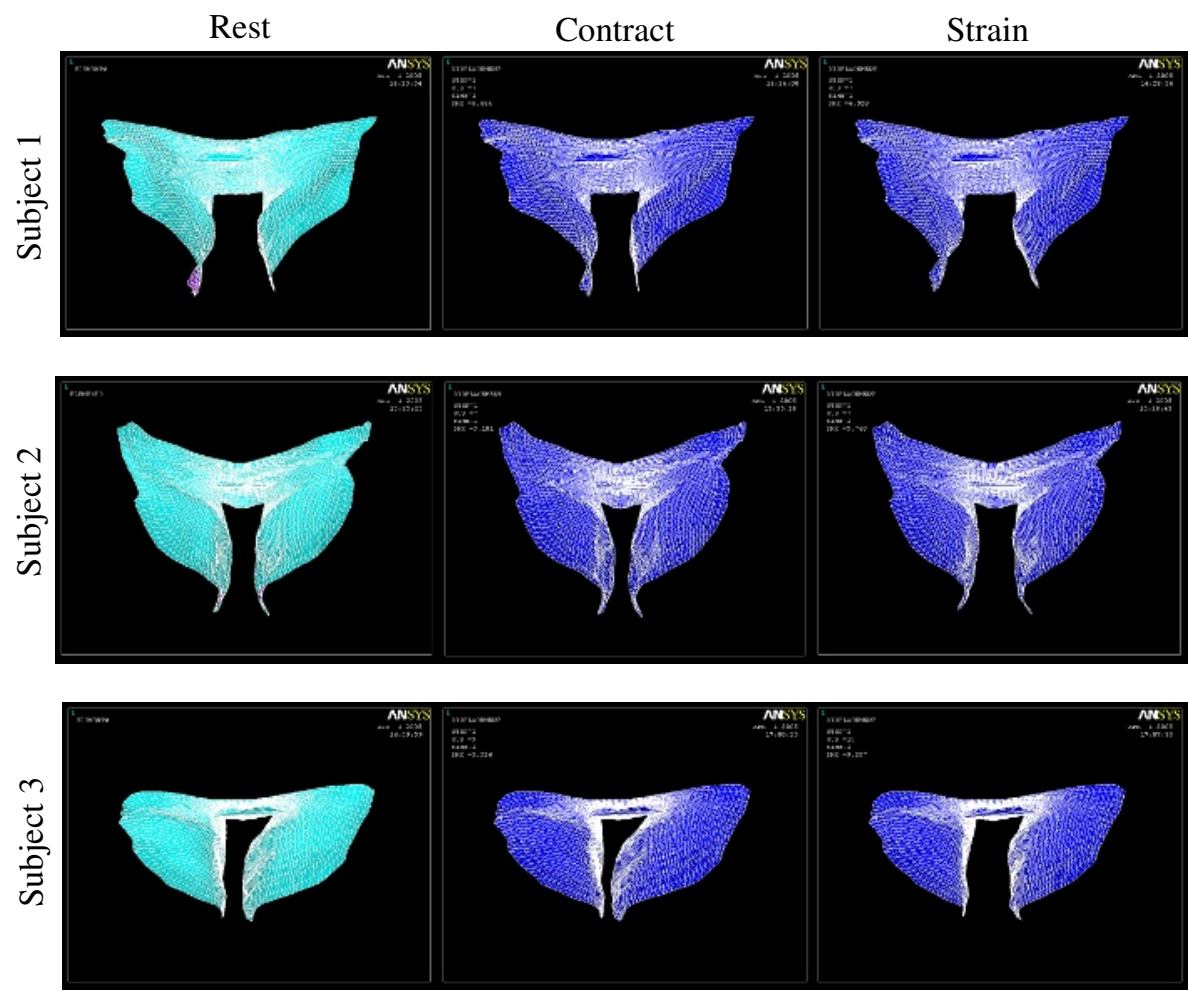

Fig. 1. The morphological changes of the levator anis for the three subjects simulated during different levels of stress: rest, contraction and strain

In Figure 3, we examine the von Mises stress in the mid-coronal plane. The graph in Figure $3 b$ shows that greater stress is located in the anterior rather than the posterior of the levator ani. This is to be expected since this area bears most of the pressure and is responsible for straightening of the anal canal, which is required during defecation.

To assess the reliability of the model, we have evaluated the change in maximal von Mises stress when the thickness of the model is changed. The levator ani surface was constructed with equal thickness at each node. In this case, the average thickness (rounded to $3 \mathrm{~mm}$ ) was used and the results are summarised in Table 1. It is evident that small variation in thickness does not result in a large change in the stress values.

To illustrate the convergence behaviour of the model, the reaction force at each time-step is plotted in Figure 4, where the load was applied in substeps from time 0 to 1 . 

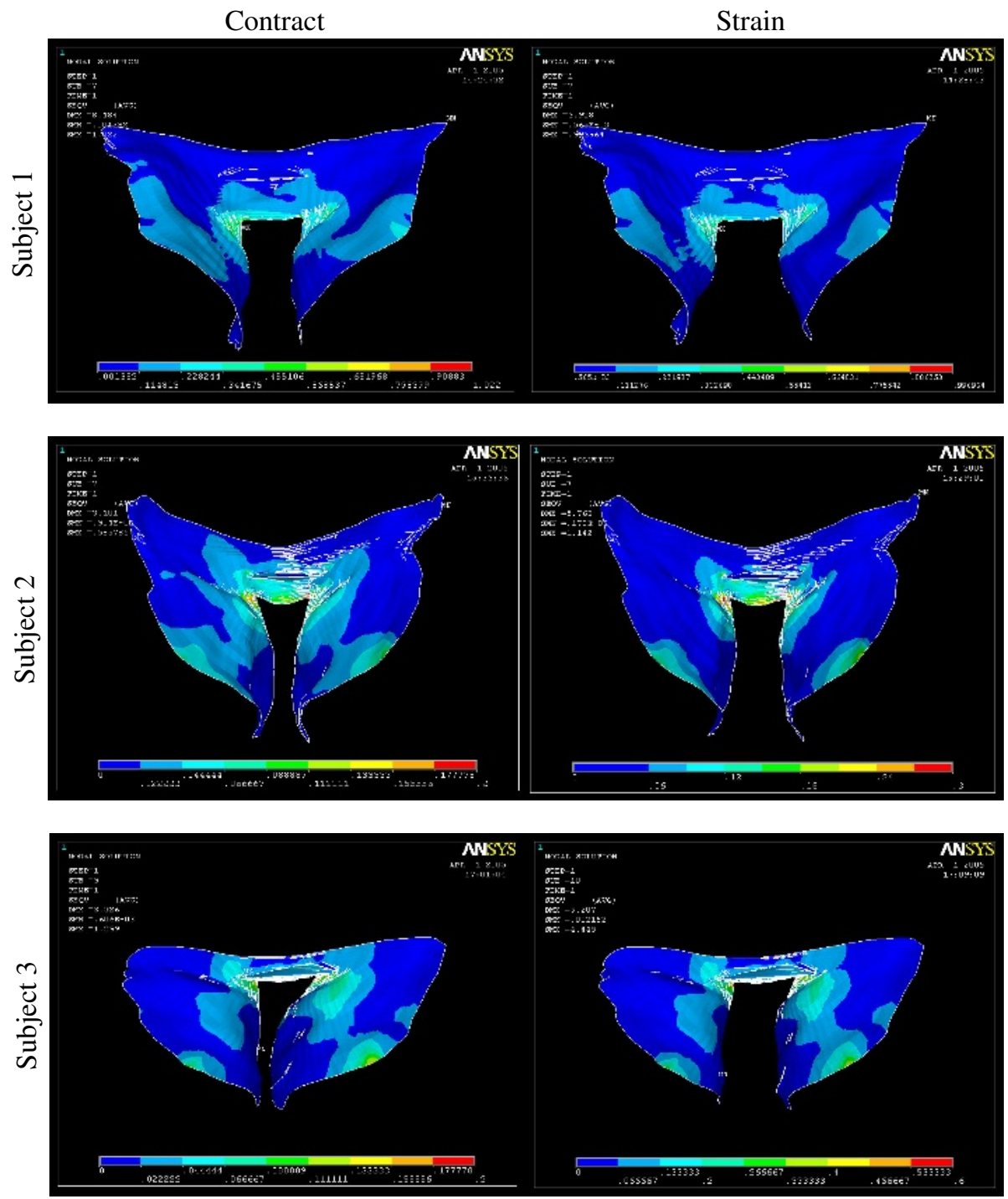

Fig. 2. The von Mises stress for each surface during contraction and strain

Table 1. Changes in the maximal von Mises stress when thickness is varied

\begin{tabular}{ccc}
\hline Thickness & Maximal von Mises stress & Percent change \\
\hline$+10 \%$ & 0.427622 & $7.68 \%$ \\
\hline$+5 \%$ & 0.445193 & $3.89 \%$ \\
\hline $3 m m$ & 0.463207 & - \\
$-5 \%$ & 0.481528 & $3.96 \%$ \\
\hline$-10 \%$ & 0.499974 & $7.94 \%$ \\
\hline
\end{tabular}



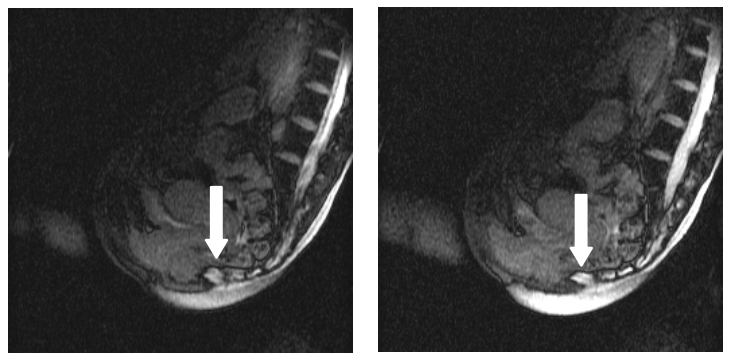

(a)

\section{von Mises Stress}

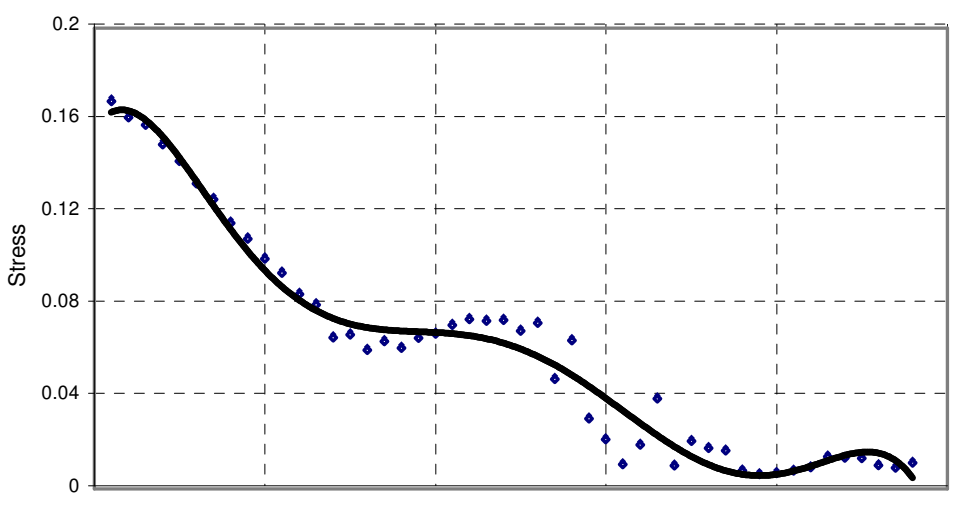

Elements from anterior to posterior on mid-coronal plane

(b)

Fig. 3. (a) Mid-Coronal MR imagrs of the pelvic floor with th levator ani (white arrow) in at rest (left) and at maximal strain dc wnwards (right) and $(b)$ the corresponding von Mises stress in the elements of the modelled levator ani lying on the mid-coronal plane

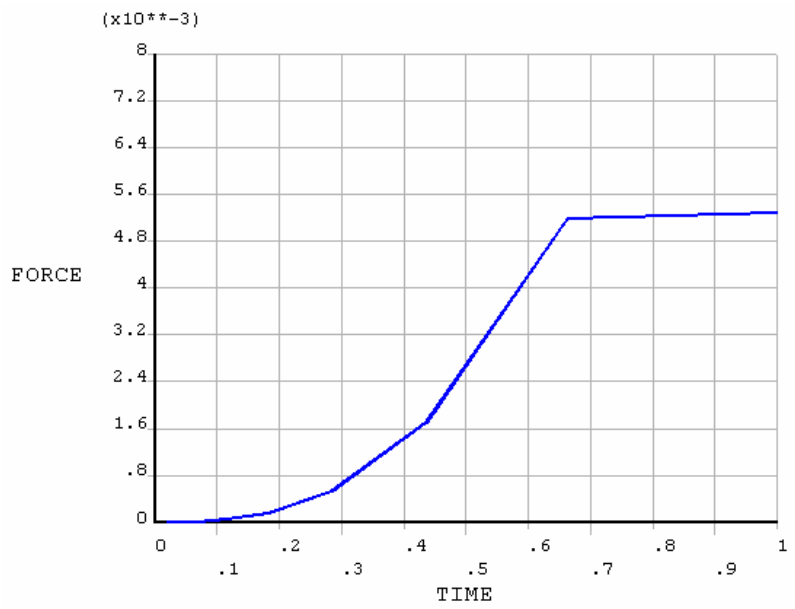

Fig. 4. Convergence behaviour of the L2 norm of the force over time 


\section{Discussion and Conclusions}

The FEM simulation based on patient specific data has shown that stress occurs mostly in the pubococcygeus and puborectalis. The associated morphological variation corresponds well with existing clinical findings as well as current work on anatomical modelling of the levator ani. We have shown quantitatively that the support to the rectum is affected most and to our knowledge, this is the first subjectspecific FEM study of the levator ani with data from dynamic MR images under natural physiological stress conditions. It is worth noting that the assessment of the accuracy of the current method is limited to evaluating the internal consistency and convergence behaviour of the model in addition to its sensitivity to potential morphological errors. In practice, in vivo quantitative validation of the model is difficult without the use of invasive means. Nevertheless, it is possible to indirectly gauge the strength of the levator ani by using vagina perineometer pressure measurements with a balloon catheter connected to a pressure gauge to determine the amount of squeeze by the pelvic floor muscles. The method, however, can be too intrusive to nulliparous subjects. In conclusion, the use of subject-specific FEM allows an improved understanding of the dynamics of a levator ani. Our results have shown consistent behaviour of the pelvic floor in normal subjects during both contraction and strain. This provides information that would not normally be available from traditional imaging techniques, thus permitting its practical use in real clinical settings.

Acknowledgements. The authors would like to thank W. Caspersz, C. Geddes, W. Gedroyc and the rest of the team at Interventional Magnetic Resonance in St Mary's Hospital, London, for their assistance in MR image acquisition. We also thank Dr Xiao Yun $\mathrm{Xu}$ for her help with finite element analysis.

\section{References}

1. Healy, J.C., Halligan, S., Reznek, R.H., Watson, S., Phillips, R.K.S.,Armstrong, P.: Patterns of Prolapse in Women with Symptoms of Pelvic Floor Weakness: Assessment with MR Imaging. Radiology. Vol 203(1). (1997) 77-81.

2. Fielding, J.R., Hoyte, L.,Schierlitz, L.: MR Imaging of Pelvic Floor Relaxation. Journal of Women's Imaging. Vol 2. (2000) 82-87.

3. Hoyte, L., Schierlitz, L., Zou, K., Flesh, G.,Fielding, J.R.: Two- and 3-dimensional MRI comparison of levator ani structure, volume, and integrity in women with stress incontinence and prolapse. American Journal of Obstetrics and Gynecology. Vol 185(1). (2001) 11-19.

4. Hoyte, L., Jakab, M., Warfield, S.K., Shott, S., Flesh, G.,Fielding, J.R.: Levator ani thickness variations in symptomatic and asymptomatic women using magnetic resonancebased 3-dimensional color mapping. American Journal of Obstetrics and Gynecology. Vol 191(3). (2004) 856-61.

5. Lee, S.-L., Horkaew, P., Darzi, A.,Yang, G.-Z.: Optimal Scan Planning with Statistical Shape Modelling of the Levator Ani. MICCAI, Montreal, Canada (2003). Lecture Notes in Computer Science, Springer, Vol 2878. 714-721. 
6. Lee, S.-L., Horkaew, P., Darzi, A.,Yang, G.-Z.: Statistical Shape Modelling of the Levator Ani with Thickness Variation. MICCAI, St Malo, France (2004). Lecture Notes in Computer Science, Springer, Vol 3216. 258-265.

7. Pathmanathan, P., Gavaghan, D., Whiteley, J., Brady, M., Nash, M., Nielsen, P., Rajagopal, V.: Predicting Tumour Location by Simulating Large Deformations of the Breast Using a 3D Finite Element Model and Nonlinear Elasticity. MICCAI, St Malo, France (2004). Lecture Notes in Computer Science, Springer, Vol 3217. 217-224.

8. d'Aulignac, D., Martins, J.,Pires, E.: Physical Modeling of the Pelvic Floor Muscles using Shell Elements. European Congress on Computational Methods in Applied Sciences and Engineering, Jyvaskyla (2004).

9. Janda, S., van der Helm, F.C.T.,de Blok, S.B.: Measuring morphological parameters of the pelvic floor for finite element modelling purposes. Journal of Biomechanics. Vol 36 (2003) 749-757.

10. Janda, S., van der Helm, F.C.T.,de Blok, S.B.: Measuring material parameters of the pelvic floor for FE modelling purposes. Proceedings Dutch Annual Conference on BioMedical Engineering, Papendal, NL (2002).

11. Janda, S., van der Helm, F.C.T.,de Blok, S.B.: Biomechanical analysis of the pelvic floor muscles: Constitutive and FE modelling. Dutch Annual Conference on BioMedical Engineering, Papendal, NL (2003).

12. Gerard, J., Wilhelms-Tricarico, R., Perrier, P.,Payan, Y.: A 3D dynamical biomechanical tongue model to study speech motor control. Recent Research Developments in Biomechanics. (2003) 49-64.

13. Chabanas, M., Payan, Y., Marecaux, C., Swider, P.,Boutault, F.: Comparison of linear and non-linear soft tissue models with post-operative CT scan in maxillofacial surgery. International Symposium on Medical Simulation, Cambridge, Massachusetts (2004). Vol 3078. 19-27.

14. Horkaew, P.,Yang, G.-Z.: Optimal Deformable Surface Models for 3D Medical Image Analysis. IPMI, Ambleside, UK (2003). Lecture Notes in Computer Science, Springer, Vol 2732. 13-24. 